\title{
Chromosome dynamics, molecular crowding, and diffusion in the interphase cell nucleus: a Monte Carlo lattice simulation study
}

\author{
Christian C. Fritsch • Jörg Langowski
}

Published online: 30 November 2010

(C) Springer Science+Business Media B.V. 2010

\begin{abstract}
Using Monte Carlo simulations, we have investigated the decondensation of chromosomes during interphase and the diffusive transport of spherical probe particles in the chromatin network. The chromatin fibers are modeled as semiflexible polymer chains on a fixed threedimensional grid, taking into account their flexibility and eventual chain crossing by the aid of topoisomerases. The network thus created will obstruct the diffusion of macromolecules. A result of our simulations is that crowding of diffusing molecules leaves the dynamics of the chromosomes and the behavior of other diffusing molecules qualitatively unaffected. Furthermore, the capability of the simulated chromatin network to trap diffusing molecules over long times is lower than that measured in microrheological experiments. Microrheology is a technique that allows to determine the viscoelastic properties of a material by the motion of embedded tracer particles. Long-time trapping requires a stiff network, as only such a network quickly responds to the diffusive fluctuations of tracers
\end{abstract}

C. C. Fritsch · J. Langowski ( $\square)$

Biophysics of Macromolecules, German Cancer Research Center

(Deutsches Krebsforschungszentrum, DKFZ),

Im Neuenheimer Feld 580, 69120

Heidelberg, Germany

e-mail: joerg.langowski@dkfz-heidelberg.de and prevents them from squeezing through meshes. A high degree of crosslinking amplifies this effect. The presence of a flexible and uncrosslinked polymer simply increases the effective viscosity sensed by tracer particles. The diffusion of tracers in our simulations reveals rather viscous than elastic chromatin networks, suggesting that chromatin alone cannot account for the high elasticity of the cell nucleus.

Keywords Chromosome dynamics • Molecular crowding • Diffusion
Abbreviations
MC Monte-Carlo
MSD mean squared displacement

\section{Introduction}

The structure of the chromatin fiber, in particular its packing topology in the cell nucleus, is critical for genome function. Gene activity is regulated by the compaction state of chromatin; DNA accessibility is determined both by the local wrapping of the DNA around the histone core and by the features of the network that is formed by the higher-order chromatin structure. Interphase chromosomes are not randomly 
intermingled polymer chains but occupy distinct "territories" in the nucleus. Accessing and handling the genetic information requires the transport of sophisticated molecular machinery to specific DNA sites, a nanomechanical process that is fundamentally influenced by the distribution and the folding behavior of chromatin.

Studying the dynamics of the chromatin fiber network is not straightforward. By following fluorescent tags with light microscopy, the compaction/decompaction, and mobility of individual loci (Müller et al. 2001) and vice versa, the mobility of fluorescent probes of various size has been studied (Bancaud et al. 2009; Dross et al. 2009; Görisch et al. 2004, 2005a; Guigas and Weiss 2008; Wachsmuth et al. 2000). However, current stateof-the-art techniques limit the resolution of such measurements to about $200 \mathrm{~nm}$, with some exceptions from single-particle tracking (Siebrasse and Kubitscheck 2009), or superresolution techniques such as STED (Klar and Hell 1999), which, however, require particular fluorophores and have rather low sensitivity. Furthermore, the interplay of chromatin fiber dynamics and transport of macromolecules has not been studied systematically, partly due to lack of suitable experimental techniques.

Generally, biomolecules are transported by passive diffusion over distances of the order of the size of the cell (Politz et al. 2003). Such diffusional transport is affected by the presence of obstacles, rendering the diffusion characteristics dependent on the length and time scale of observation. In normal diffusion, as typically observed in homogeneous solution, the mean squared displacement (MSD) $\left\langle r^{2}(t)\right\rangle$ of a molecule is proportional to the time $t$. Diffusion is called anomalous if $\left\langle r^{2}(t)\right\rangle \propto$ $t^{\alpha}$, with an anomaly parameter $\alpha \neq 1$. Obstacles typically cause $\alpha$ to become $<1$; this case is called subdiffusion (Metzler and Klafter 2000).

The observation of anomalous diffusion also depends on the length scale. On a very short scale, the walker has not yet traveled far enough to encounter many obstacles by which its walk could get obstructed. The walker "sees" almost free, homogeneous space and diffusion is nearly normal. On a larger scale, the walker senses both obstacles and free space, its environment appears inhomogeneous, and diffusion becomes anomalous. On a very large scale, obstruction effects average out, diffusion becomes normal again, and the walker "remembers" a homogeneous medium. Fluorescence correlation spectroscopy showed subdiffusion in cell nuclei (Wachsmuth et al. 2000) and chromatin-induced obstruction of larger molecules (Görisch et al. 2004, 2005a), whereas smaller molecules diffused normally and no correlation between their mobility and the density of chromatin was found (Dross et al. 2009).

The accessibility of a polymeric material to diffusing molecules primarily depends not only on the size of the molecules and the global density of the polymer matrix but also on the spatial arrangement of the matrix and on its dynamics. In a homogeneous immobile polymer network, molecules are retained if they are larger than the characteristic mesh size. At the same global density, a network with a very inhomogeneous mesh size distribution, i.e., with a tendency of the network fibers to lump together, can leave space for channels that allow large molecules to travel greater distances.

To assess the importance of obstructional effects in cell nuclear transport, we need to know the occupation of the nuclear space by chromatin and other macromolecules. Using a combination of fluorescence correlation spectroscopy and confocal imaging, we determined earlier that chromatin occupies about $10 \%$ of the nuclear space (Weidemann et al. 2003). However, the overall occupation of cellular compartments by macromolecules is between $5 \%$ and $40 \%$ (Ellis and Minton 2003), i.e., the volume of the cell nucleus contains up to $30 \%$ macromolecules other than chromatin. Many studies have looked at molecular crowding as the origin of anomalous diffusion (for a review, e.g., see Dix and Verkman 2008). In the cell nucleus, however, the precise contributions of the different molecular species to diffusional obstruction and anomaly and therefore the relevance of anomalous diffusion to genomic function in general are not clearly understood.

In previous papers, we developed a coarsegrained lattice model for the chromatin fiber as a very efficient tool to generate thermodynamic equilibrium conformations of the chromatin chain 
with varying persistence lengths and densities. This allowed us to study the diffusional properties of macromolecules in the chromatin network (Wedemeier et al. 2007), the effect of binding and sliding of proteins on DNA (Wedemeier et al. 2008), and the squeezing of proteins through dense dynamic chromatin networks (Wedemeier et al. 2009). It offers the advantage that physically consistent chain conformations can be generated in a computationally inexpensive way, since only local interactions have to be considered. In previous work on the effect of specific folding topologies on diffusion (Fritsch and Langowski 2010), we concentrated on the purely geometric obstruction effect of chromatin, assuming the simulated chromatin chain immobile and affecting diffusion only via steric volume exclusion. There we succeeded in developing a theory for the spatial arrangement of a heterogeneous polymer network in terms of correlations and derived a measure for its "clumpiness" and how this affects macromolecular transport. Simultaneously, this model allowed us to study the effect of loopinduced compartmentalization, a concept that has been discussed for some time (Bohn et al. 2007; Cremer et al. 1996; Münkel and Langowski 1998), and to compare the simulation results quantitatively with the recent intrachromosomal interaction data measured by Lieberman-Aiden et al. (2009). However, the combined effects of chromatin chain mobility, specific chromatin folding, and of crowding by other diffusing macromolecules were not included in our earlier work.

Here we have generated dynamic chromatin networks with characteristic interphase topologies and analyzed the diffusion of macromolecules in crowded and uncrowded conditions. We show that including chain dynamics allows larger macromolecules to penetrate the network, and we analyze the effect of local chain flexibility and possible self-crossing of the chromatin chain (e.g., induced by type II topoisomerases). We verify the relaxation time scales for interphase chromosomes recently estimated by Rosa and Everaers (2008) by a continuum model and show that molecular crowding by diffusing macromolecules can lead to anomalous diffusion but to a much lesser degree than crowding by the chro- matin network. We also find that the stiffness of the network probed with diffusing particles is much lower than detected earlier in microrheology experiments (Tseng et al. 2004).

\section{Chromatin model}

Lattice discretization of the chromatin fiber

Our simulation approximates interphase chromatin by a semi-flexible chain polymer (Rubinstein and Colby 2003). Each bond between the segments of this chain polymer is represented by a site on a Cartesian lattice. In order to compose a connected chain, a certain distance between subsequent bonds has to be maintained. In our simulations, we allow the bond lengths $l=1, \sqrt{2}$, and $\sqrt{3}$ (in lattice units). The bending angle $\theta_{i}$ at the $i$-th bond is drawn by the two segment vectors $\vec{l}_{i-1}$ and $\vec{l}_{i}$. The bending rigidity of chromatin is modeled by harmonic potential functions of $\theta_{i}$ which are installed at each bond, yielding the total internal energy of the chain

$E=\frac{g}{2} \sum_{i=1}^{N-2} \theta_{i}{ }^{2}$,

where $N$ and $g$ denote the number of chain bonds and the bending rigidity constant in units of $k_{B} T$ (Fritsch and Langowski 2010; Wedemeier et al. 2007). The persistence length $L_{p}$ of the chain is the characteristic length over which the chain behaves approximately like a rigid rod and is directly related to $g$ (for a detailed derivation of the connection between the persistence length and the internal bending rigidity on the Cartesian lattice, see Fritsch and Langowski 2010). To account for steric volume exclusion (self-avoidance), the occupation of a lattice site by two or more distinct chain bonds is prohibited. For modeling a phantom chain, this rule is suspended to facilitate chain crossings.

The decondensation of a chromosome during interphase is simulated by a gradual equilibration of an initial metaphase-like conformation of the chromatin fiber. For this equilibration, a Metropolis Monte Carlo (MC) procedure is performed 
during which single MC steps randomly alter the positions of chain bonds on the lattice (Carmesin and Kremer 1988; Metropolis et al. 1953). In this procedure, the internal bending rigidity is explicitly accounted for, and after a sufficiently large number of steps, the initial condensed chromatin conformation has transformed into a statistically more favorable decondensed interphase conformation (see also Fritsch and Langowski 2010).

Lattice random walks model molecular diffusion

Diffusing molecules are represented by quasispherical lattice bodies. Their motion is simulated by random walks on the lattice. At a single step of the walk, one of the six nearest neighbor sites of the center of the molecule is picked randomly as a candidate for a new position of the center. The diffusing molecule must not occupy the same space as the chromatin chain or other diffusing molecules. If the displacement to the candidate position results in a double occupation of a lattice site, which means that the molecule overlaps with an obstacle, the displacement is not performed and the molecule stays on its site during this step (see Fig. 1a-c). If no overlap occurs, the molecule is displaced, and the new position is recorded (see Fig. 1d-f). The MSD of the molecule is measured as a function of time, averaged over 1,000 single trajectories.

\section{Conversion of lattice units to physical units}

For both the monomer displacement during the Metropolis Monte Carlo procedure and the displacements of the randomly walking molecules, the lattice units and MC time steps can be converted to physical units. Chromatin is often associated with a "30-nm fiber". As a cylinder, its volume is $V_{\text {cyl }}=\pi d^{2} L / 4$, where $d$ is the diameter and $L$ is length of the chromatin fiber. For our simulations, we consider chromatin a chain of beads, and then its volume is $V_{\text {cob }}=N 4 \pi R_{0}^{3} / 3$, where $N$ is the number of beads and $R_{0}$ is the bead radius. For both models to be equivalent, we require the same length $\left(L=2 N R_{0}\right)$ and the same volume, and then the bead radius is $R_{0}=$ $(3 / 8)^{1 / 2} d=18 \mathrm{~nm}$. Since each bead corresponds

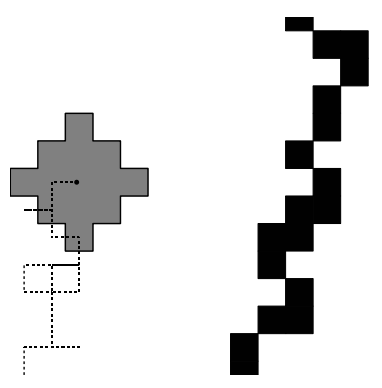

(a)

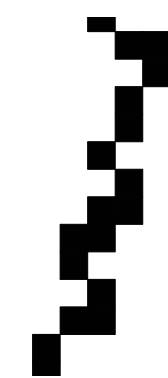

(c)

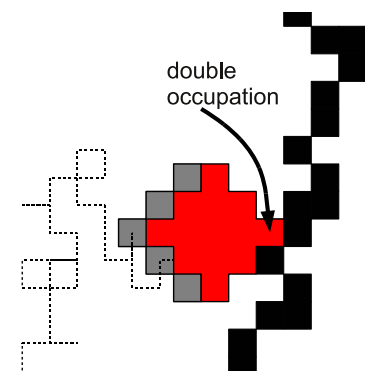

(e)

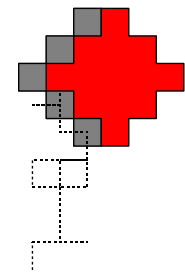

(b)

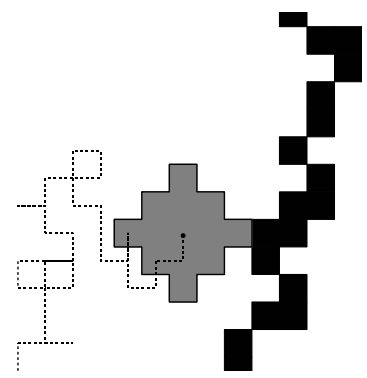

(d)

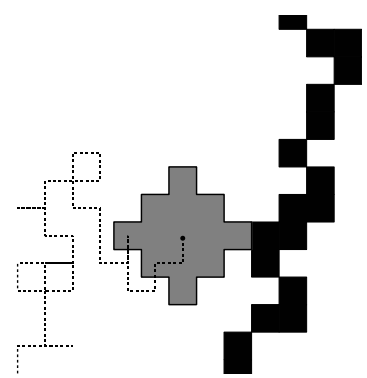

(f)
Fig. 1 Two-dimensional illustration of molecule interacting with an obstacle while performing a random walk. a A molecule represented by a quasi-spherical lattice body (gray) in the proximity of an obstacle (black chain). The trajectory of the molecule is indicated by the dashed black lattice curve. b The molecule attempts a random walk step that displaces the molecule to a position on which it does not overlap with the obstacle (red area). This step is permissible. $\mathbf{c}$ The step has been executed and the molecule has moved on. d At some other point in the random walk, the molecules has approached the obstacle. e The molecule attempts a random walk step that displaces the molecule to a position on which it does overlap with the obstacle. This step is forbidden. f The step has been rejected and the molecule remains on its site

to one lattice site, we define the lattice constant ${ }^{1}$ $a=36 \mathrm{~nm}$.

${ }^{1}$ The lattice constant is the physical length that corresponds to the spacing of the lattice. 
Now the Monte Carlo steps can be converted to a physical time scale considering the monomer relaxation time of a dynamic polymer: In a polymer chain with the monomer size $b$, a single monomer requires the time $\tau_{0} \approx \eta b^{3} /\left(k_{B} T\right)$ to travel a distance of the order of its own size (Rubinstein and Colby 2003). Up to this time scale, it does not feel the connection to other beads in the polymer, and the distance covered within a single MC step is always $r=36 \mathrm{~nm}$. In water $\left(\eta=10^{-3} \mathrm{Nsm}^{-2}\right.$ at $\left.T=300 \mathrm{~K}\right)$ and for monomers with $b=36 \mathrm{~nm}$, we get $\tau_{0}=12 \mu \mathrm{s}$, which we define as the duration of a chain monomer displacement as well as the duration of a random walk displacement of a molecule with radius $R_{0}=$ $18 \mathrm{~nm}$. In order to obtain the step duration $\tau$ of molecules with radii $R>R_{0}, \tau_{0}$ is to be scaled according to the Stokes-Einstein relation $D=$ $k_{B} T /(6 \pi \eta R)=r^{2} /(6 \tau)$, and we get $\tau=\tau_{0} R / R_{0}$.

\section{Simulation of interphase chromosomes}

\section{Creating realistic chromosomes}

In order to provide for a realistic simulation of molecular diffusion and chromatin dynamics, the lattice model must reproduce precisely the degree of obstruction that diffusing molecules or chromatin fibers would experience in a corresponding continuum model. In a series of test sim- ulations, we use the concept of lattice refinement to extrapolate the continuum as the limiting case of an infinitely refined lattice. From that we deduce the correct lattice occupation for the main simulation of realistic chromosomes on an unrefined lattice.

A single site on a three-dimensional Cartesian lattice with lattice constant $a$ represents a cube with edge length $a$ rather than just a point in space. On a $10 \times 10 \times 10$ lattice with $a=36 \mathrm{~nm}$, for instance, a site corresponds to a physical cube with edge length $36 \mathrm{~nm}$ (see Fig. 2, first panel from the left). Refinement of the lattice by a factor $\xi$ transforms it into a $(\xi \times 10) \times(\xi \times 10) \times(\xi \times 10)$ lattice. The edge length of the cube is kept at $36 \mathrm{~nm}$, which transforms this cube into a $\xi \times \xi \times$ $\xi$ cube and reduces the lattice constant to $a_{\xi}=$ $36 \mathrm{~nm} / \xi$ (in Fig. 2, steps from the second panel to the fourth panel and from the third to the fourth).

In the previous section, we have considered chromatin a chain of beads, not cubes. By replacing the refined cube with a quasi-spherical lattice body, a bead can be approximated. The better the approximation, the larger the $\xi$, and as $\xi$ approaches infinity, this lattice body becomes a perfect sphere. Figure 3 illustrates the refinement of the lattice and the increasing quality of a sphere approximation with rising $\xi$.

Lattice refinement as illustrated drastically increases the computation effort for simulating the diffusive motion of a chain monomer, since

refinement by factor $\xi=5$, yielding a $50 \times 50 \times 50$ lattice

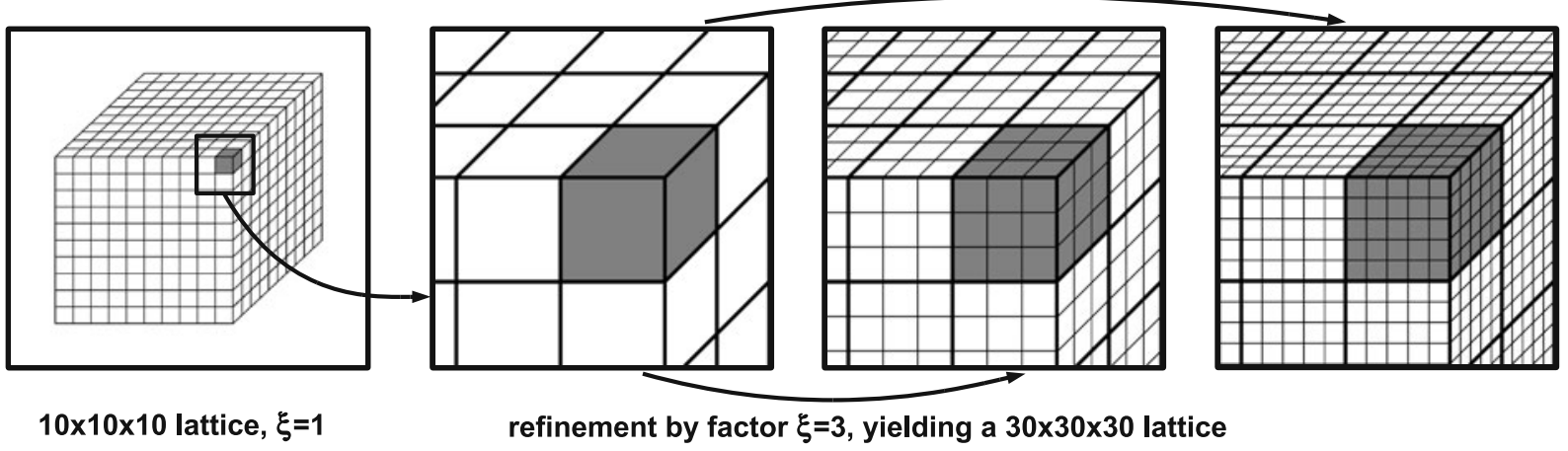

Fig. 2 Refinement of the simulation lattice. The volume of the space that is represented by the lattice remains the same after a refinement. The edge length of the gray cube is $36 \mathrm{~nm}$ throughout. With increasing the refinement factor $\xi$, the physical length corresponding to the spacing of the lattice, $a_{\xi}$, is reduced. This allows a representation of physical objects with increased resolution 

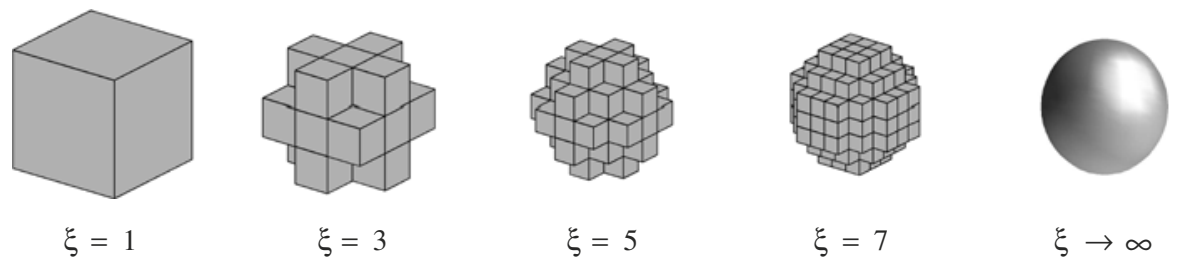

Fig. 3 Refinement of a lattice sphere. With increasing refinement factor $\xi$, the approximation of a sphere by a quasispherical lattice body can be improved

every single lattice site that is part of a monomer has to be moved during a displacement. Furthermore, every single site has to be checked whether a displacement of the monomer leads to a double occupation. Thus, we checked how this refinement could be avoided for simulating a whole chromosome and which parameters allow us to approximate a chain of smooth beads by a single-site $(\xi=1)$ lattice chain.

The typical volume fraction of chromatin in the cell nucleus is about $10 \%$ (Weidemann et al. 2003). The ratio of the volumes of a cube at $\xi=1$ and the sphere at $\xi \rightarrow \infty$ is $a^{3} /\left(4 \pi(0.5 a)^{3} / 3\right) \approx 2$. Therefore, in order to simulate a chain of beads that occupies $10 \%$ of a predefined space, the corresponding chain of lattice sites would have to occupy approximately $20 \%$ of the lattice. With a simulation of crowding diffusants, we checked if the obstruction effect on diffusing molecules in these two problems was really comparable. At lattice refinements $\xi=1,3,5,7,9,11$, and 13 on $(\xi \times 10) \times(\xi \times 10) \times(\xi \times 10)$ lattices, we calculated the average diffusion coefficient $D$ of 200 simultaneously diffusing lattice spheres. We defined the reduced diffusion coefficient $D / D_{0}$, where $D_{0}$ denotes the diffusion coefficient of a freely diffusing sphere in solution. In Fig. 4, the reduced diffusion coefficients are shown in dependence of the lattice refinement. As $\xi$ increases, $D / D_{0}$ approaches a constant value of approximately 0.76 , which we regard as the continuum limit of the refinement and therefore as the reduced diffusion coefficient of a perfect sphere. The value of $D / D_{0}$ at $\xi=1$ is 0.78 , which deviates only $2.5 \%$ from the extrapolated limit. The fraction of occupied lattice sites (also shown in Fig. 4) drops from 0.2 at $\xi=1$ to approximately 0.1 at large values of $\xi$. Hence, we validated the substitution of beads with lattice sites regarding the obstruction effect in a diffusant crowding situation. In another test simulation, we also verified that this substitution is valid if the lattice sites are arranged as a chain.

In the simulation of the decondensation of 100Mbp chromosomes during interphase, we used three different models of the chromatin fiber: (a) a self-avoiding chain without internal bending rigidity, (b) a self-avoiding chain with an internal bending rigidity corresponding to a persistence length $L_{p}=300 \mathrm{~nm}$, and (c) a phantom chain with $L_{p}=300 \mathrm{~nm}$. Assuming a chromatin packing ratio of $100 \mathrm{bp} / \mathrm{nm}$, the simulated chromatin fiber is $1 \mathrm{~mm}$ long, and with an average bond length corresponding to $50 \mathrm{~nm}$, which we had obtained from previous test simulations, the lattice chain

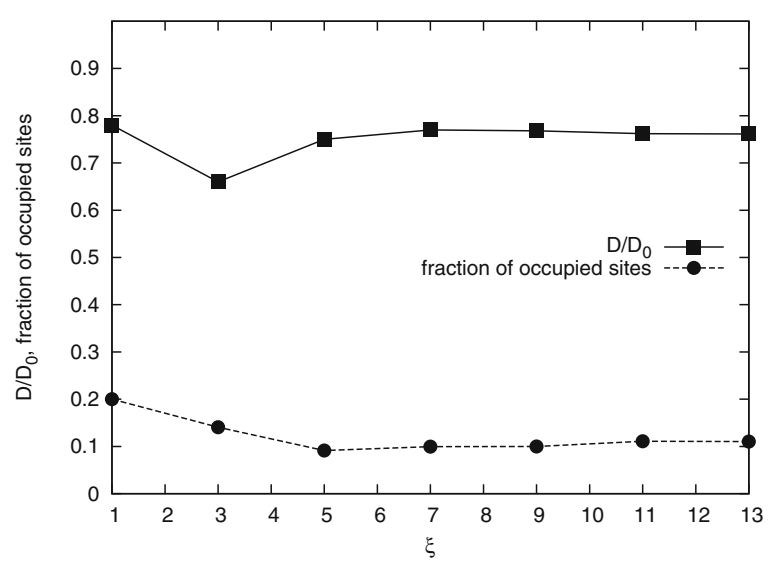

Fig. 4 Approaching the continuum limit with lattice refinements. The data show the reduced diffusion coefficients $D / D_{0}$ (squares) of 200 simultaneously diffusing lattice spheres and the fraction of occupied sites (circles) at different refinements $\xi$. As $\xi$ increases, both the reduced diffusion coefficient and the fraction of occupied sites approach a constant value, which allows to extrapolate the continuum limit for diffusing perfect spheres 
was to be composed of $N=20,240$ sites. In order to make a chain of 20,240 sites occupy $20 \%$ of the simulation cube, the edge length had to be 46 lattice units. The initial conformation of our lattice chromatin (see Fig. $5 d$ ) on the $46 \times 46 \times 46$ lattice consists of stacked rosette-like chromatin disks and resembles a metaphase chromosome. It has a diameter of $600 \mathrm{~nm}$ and a length of $4.4 \mu \mathrm{m}$. For each of the chromatin models, we applied the Monte Carlo equilibration procedure on ten independent initial conformations. During every time step, 20,240 single bonds of a conformation were randomly picked for an eventual displacement. Every initial conformation ran through $10^{7}$ time steps ( $\approx 120$ s real time), and then we evaluated the resulting end conformation. Typical end conformations for the three models and the initial conformation are shown in Fig. 5.

To avoid confinement artifacts we used periodic boundaries. If a chain monomer (or a diffusing molecule) leaves the $46 \times 46 \times 46$ lattice during an MC step at one boundary, it simply re-enters the lattice through the opposite. That way chromosomes can expand beyond the actual extent of the lattice and the nuclear space becomes virtually infinite. At the same time, the overall occupation density of the lattice is conserved.

\section{Decondensation dynamics}

During the equilibration, we recorded the average MSD of single monomers, the MSD of the centerof-mass of the chain, and its gyration radius. The time-dependent MSD of the monomers characterizes the fastest relaxation mode of the chain and reflects the physical properties of the chain on certain time scales. In a chain polymer that is not subject to hydrodynamic forces, internal bending rigidity, or external stress, the MSD of a
Fig. 5 Simulated 100-Mbp chromosomes after $120 \mathrm{~s}$ of decondensation during interphase. The tripods indicate the size of the underlying periodic lattice (arrow length $\approx 1.6 \mu \mathrm{m})$. a A self-avoiding chain without internal bending rigidity, b a self-avoiding chain with a persistence length $L_{p}=300 \mathrm{~nm}$, c a phantom chain with $L_{p}=300 \mathrm{~nm}$, and d the initial metaphase conformation, which is composed of stacked disks. The inset shows the arrangement of lattice sites for such a disk

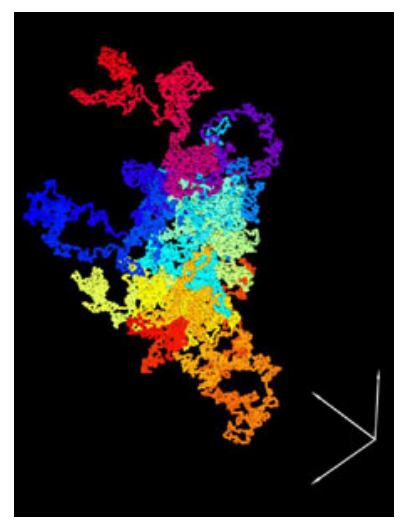

(a)

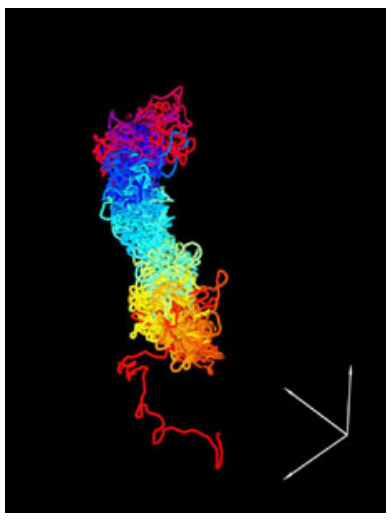

(b)

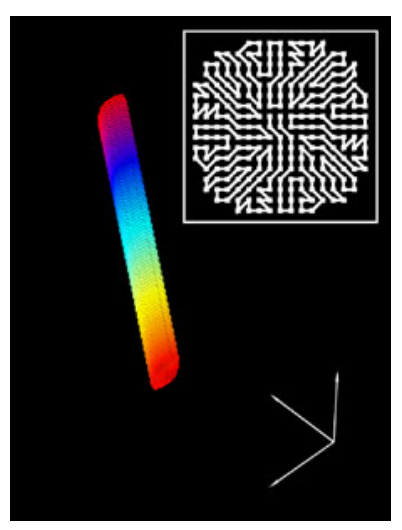

(d)

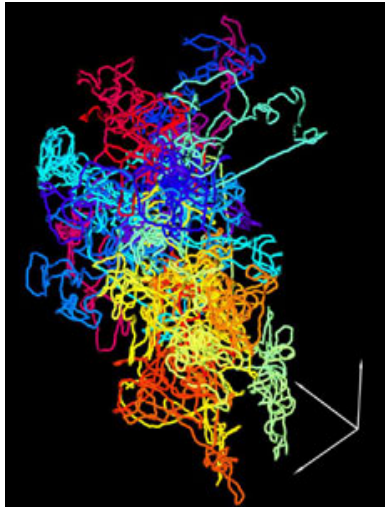

(c) 
monomer is proportional to $t^{0.5}$ up to the Rouse time $\tau_{R}$ at which the whole polymer has traveled a distance of its own size (Doi and Edwards 1986). For times longer than $\tau_{R}$, its MSD is proportional to $t$ and coincides with that of the center-of-mass. In a semi-flexible chain, the MSD of a monomer is proportional to $t^{0.75}$ on time scales on which the local motion of semi-flexible chain is dominated by its internal bending rigidity (Farge and Maggs 1993). If the polymer is very dense, the local motion is governed by the self-entanglement of the polymer and the MSD is proportional to $t^{0.25}$ (Doi and Edwards 1986).

Figure 6 shows the time-dependent MSD of the monomers during the decondensation. For the self-avoiding chain without internal bending rigidity (model A), the MSD of the center-ofmass is also shown in the inset. In MC simulations with ideal lattice chains, Rouse dynamics $\left(\left\langle r^{2}\right\rangle \sim t^{0.5}\right)$ could be reproduced in agreement with theoretical predictions, which proved the validity of the lattice MC approach. Since a metaphase chromosome poses a very specific nonequilibrium conformation of chromatin, we conduct non-equilibrium statistics, and the MSD of

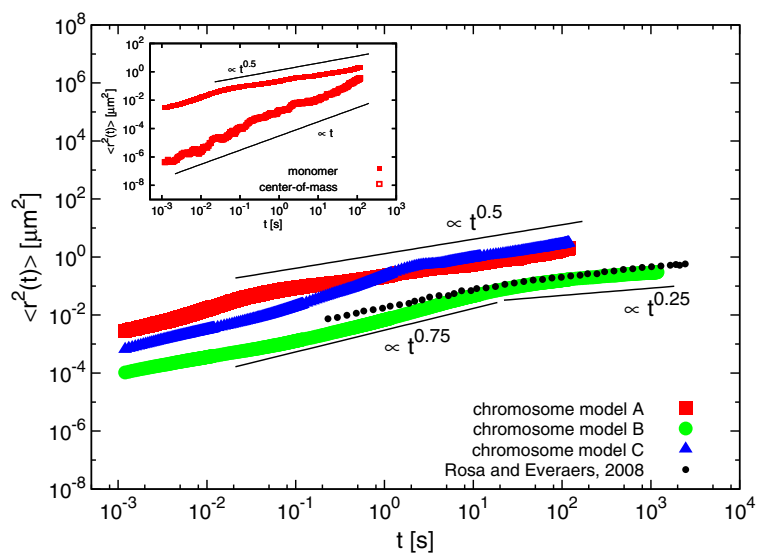

Fig. 6 Relaxation modes of the simulated decondensing chromosomes. The outer plot shows the mean squared displacement $\left\langle r^{2}(t)\right\rangle$ of the monomer relaxation modes versus time $t$. Red squares: self-avoiding chain polymer without internal bending rigidity (model A), green circles: self-avoiding chain polymer with a persistence length $L_{p}=$ $300 \mathrm{~nm}$ (model B), blue triangles: phantom chain polymer with $L_{p}=300 \mathrm{~nm}$ (model C), black dots: result from Rosa and Everaers 2008. In the inset, the MSD of the monomers (full squares) and of the center-of-mass (empty squares) are shown the monomers in our model A chromosome does not precisely obey Rouse dynamics. Nevertheless, we can estimate its relaxation time $\tau_{R}$ by intersecting the extrapolated MSD curves of the monomers and the center-of-mass (extrapolations not shown in Fig. 6). From that we obtain $\tau_{R} \approx$ $1,000 \mathrm{~s}$, which is well within the duration of interphase in most human cells.

The MSD of the self-avoiding chain with persistence length $300 \mathrm{~nm}$ (model B) exhibits a semiflexible polymer regime up to about $10 \mathrm{~s}$ and entanglement on longer times scale. We have equilibrated the conformations of model B ten times as long as the others to make the onset of the entanglement regime visible. In a similar study by Rosa and Everaers (2008), Brownian dynamics simulations were used to investigate the decondensation of 100-Mbp chromosomes. Their result for simulated chromosomes, which are comparable to our model B chains, is represented by the black dots in Fig. 6, agreeing well with our result obtained by Monte Carlo simulations. The intersection of the extrapolated MSD curves of the monomers and the center-of-mass for the model B yields a relaxation time of about 100 days, which is more than the lifetime of most human cells.

Within the first second of the decondensation, the monomer relaxation mode of the phantom chain (model $\mathrm{C}$ ), which represents a chromosome with high topoisomerase II activity, ranges between those of models $\mathrm{A}$ and $\mathrm{B}$. This suggests that in the compact and unequilibrated initial conformation, a high persistence is more obstructive than self-avoidance. However, after approximately $0.1 \mathrm{~s}$, the model $\mathrm{C}$ chromosome starts to unfold rapidly, with an MSD proportional to $t^{1.08}$ until about $1 \mathrm{~s}$. Then it decondenses Rouse-like and slightly faster than the model A chromosome.

Figure 7 shows the gyration radii of the chromosomes as a function of time during their decondensation. The gyration radius of a polymer is the RMS distance of a monomer from the center-of-mass of the polymer and can be taken as a measure for the unfolding. While the rather well decondensing chromosomes of models $\mathrm{A}$ and $\mathrm{C}$ show no tendency to reach a maximum gyration radius within at least $120 \mathrm{~s}$ after the start of the decondensation, the model $\mathrm{B}$ chromosome reaches a maximum gyration radius after about 


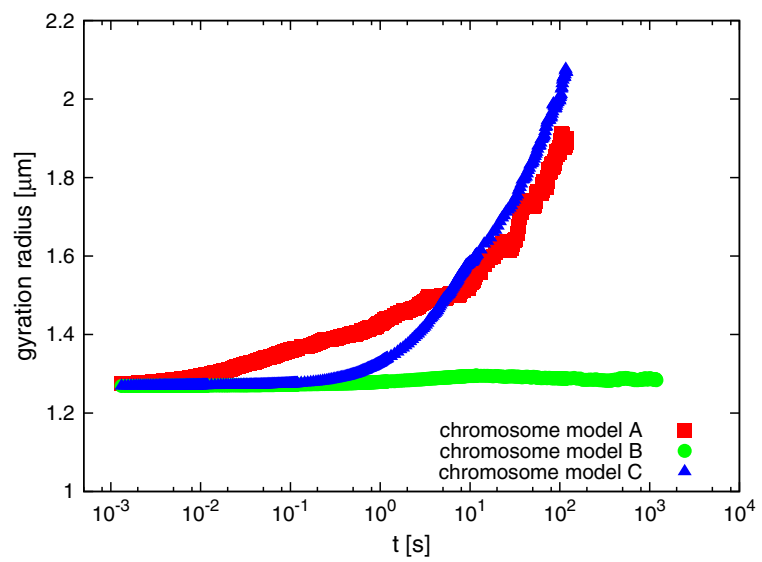

Fig. 7 Gyration radii of simulated chromosomes during their decondensation. Red squares: self-avoiding chain polymer without internal bending rigidity (model A), green circles: self-avoiding chain polymer with to a persistence length $L_{p}=300 \mathrm{~nm}$ (model B), blue triangles: phantom chain polymer with $L_{p}=300 \mathrm{~nm}$ (model C)

$10 \mathrm{~s}$ and from then on its decondensation stagnates.

\section{Chromosome compartmentalization}

The lattice model allows us to analyze to which extent chromosomes form subcompartments, i.e., sections of the chromatin fiber on which all genomic sites are spatially close. Compartmentalization can be illustrated by generating proximity maps of genomic sites and compare them with similar data recently obtained experimentally (Lieberman-Aiden et al. 2009): First, we computed the matrix $R^{*}$ of the distances $r_{i, j}^{*}$ between every pair of bonds $i$ and $j$ in the simulated chromosome. Then we averaged $R^{*}$ down to a tenfold smaller matrix $R$ in which every entry was the arithmetic average of the entries in the corresponding $10 \times 10$ submatrix of $R^{*}$. This averaging is to reduce the computation effort for the following processing of the data. From $R$ we obtained the correlation matrix $C$ in which an entry $c_{i j}$ is the Pearson correlation coefficient of the $i$-th row and the $j$-th column of $R$ :

$c_{i j}=\sum_{l=1}^{N} \frac{\left(r_{i l}-r_{i}\right)\left(r_{l j}-r_{j}\right)}{\sigma_{i} \sigma_{j}}$, where $r_{i}=\frac{1}{N} \sum_{j=1}^{N} r_{i j}$ and $\sigma_{i}=\sqrt{\frac{1}{N} \sum_{j=1}^{N}\left(r_{i}-r_{i j}\right)^{2}}$. The $c_{i j}$ express the spatial correlations of 500-nm sections of chromatin and take values between -1 and 1 , which mean mutual remoteness and closeness, respectively. The matrix $C$ can then be visualized as a map. We refer here to the proximity maps of single chromosomes as "autoproximity" maps in order to distinguish them from the proximity maps between two chromosomes.

Figure 8 shows typical auto-proximity maps of the three chromatin models after $120 \mathrm{~s}$ of decondensation (corresponding to the conformations in Fig. 5). Yellow blocks indicate the compartmentalization of numerous sections of the chromatin fiber. According to that, model A chromosomes tend to form large distinct compartments. This can be considered a consequence of the missing bending rigidity which enables the chromatin fiber to fold back on itself over short contour lengths. The crumpling of a metaphase conformation can easily be preserved this way. In contrast to that, chromatin fibers with high bending rigidity can form loops only over long contour lengths. This can in turn be suppressed by topological constraints as in model B chromosomes. Model C chromosomes, which may cross themselves, do not feel such constraints and can widely protrude. In the latter case, this results in close contacts between sections that are genomically far apart, while real compartments form to a much lower degree. The general closeness of distinct sections of chromatin is trivially fulfilled in the rather condensed model B chromosome (Fig. 8b). The dumbbell-shaped yellow area is an artifact of the statistical treatment. Due to the fact that in the condensed state central sections are generally close to other sections, the row (or column) averages $r_{i}$ of central rows are greater than those of end rows. According to Eq. 2, this results in greater $c_{i j}$ at the ends of the matrix diagonal.

\section{Chromosome territories}

Similar to the auto-proximity maps of single chromosomes, we constructed the cross-proximity maps of neighboring chromosomes, whose overlap or non-overlap can be displayed this way. The computation of the correlation matrix $C$ is 


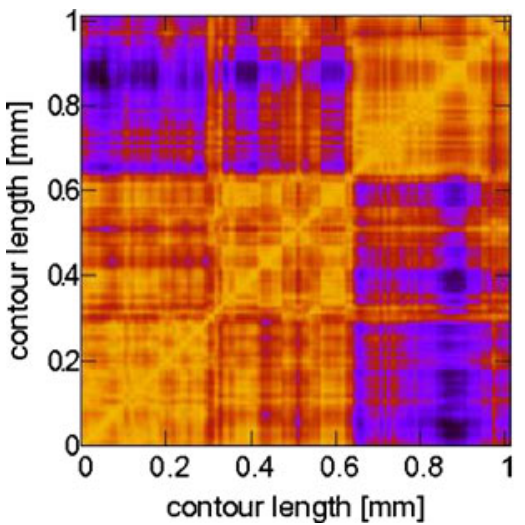

(a)

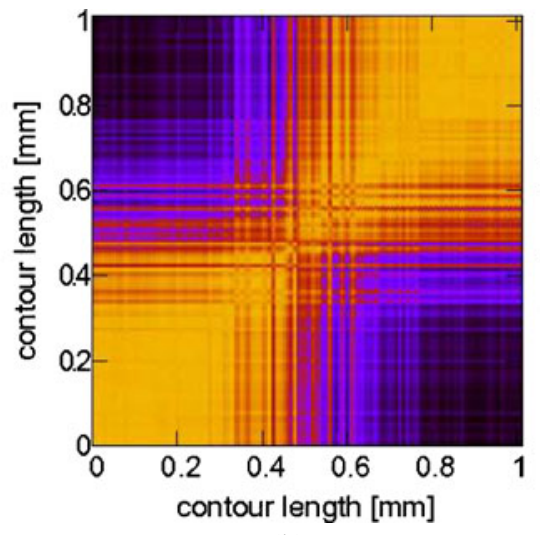

(b)

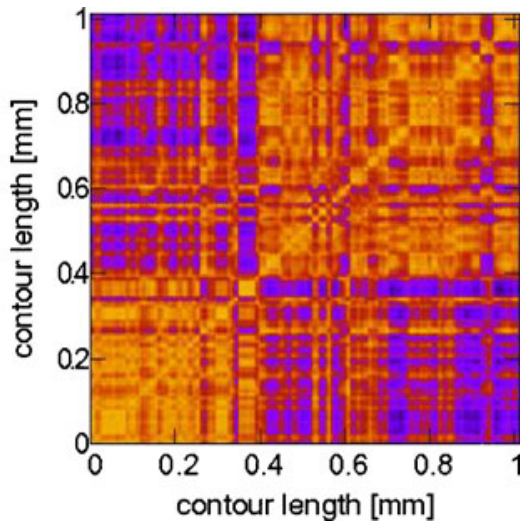

(c)
Fig. 8 Auto-proximity maps of chromosomes after $120 \mathrm{~s}$ of decondensation during interphase. The $x$-axis and the $y$-axis of the maps correspond to the contour of a single chromosome. a A self-avoiding chain polymer without internal bending rigidity, $\mathbf{b}$ a self-avoiding chain polymer with a persistence length $L_{p}=300 \mathrm{~nm}$, and $\mathbf{c}$ a phantom chain polymer with $L_{p}=300 \mathrm{~nm}$. Blue: negative spatial correlation, yellow: positive spatial correlation principally the same as for the auto-proximity maps, with the only difference that the distances $r_{i, j}^{*}$ are now measured between segments on distinct chromosomes. During the decondensation procedure, we simulated only one chromosome at once, but because of the periodic boundaries, we can construct a virtual second chromosome as an identical copy of the first, shifted by the period of the underlying lattice. The typical cross-proximity maps displayed in Fig. 8 correspond to the three chromatin conformations shown in Fig. 5.

For all three chromatin models, at least a partial intermingling of neighboring chromosomes is evident. In the maps for model $\mathrm{A}$ and particularly for model $\mathrm{C}$, an intermingling throughout the whole chromosomes occurs, and a separation into distinct chromosome territories is impossible (Fig. 9).

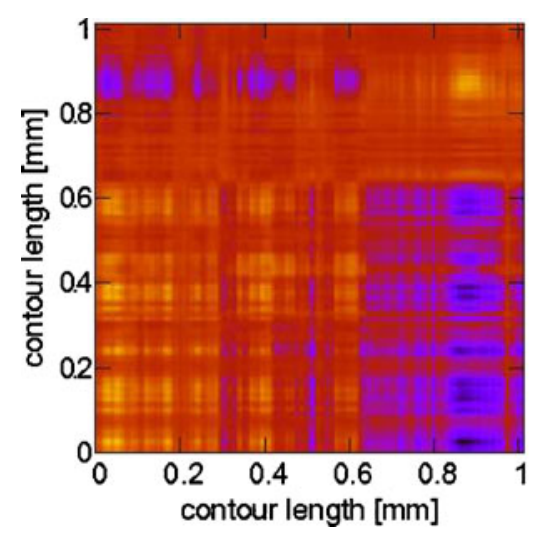

(a)

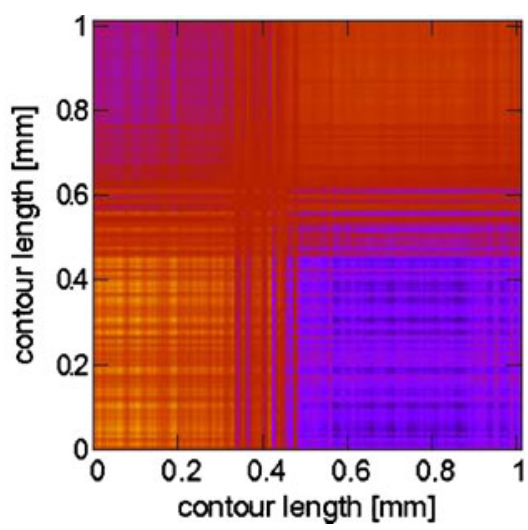

(b)

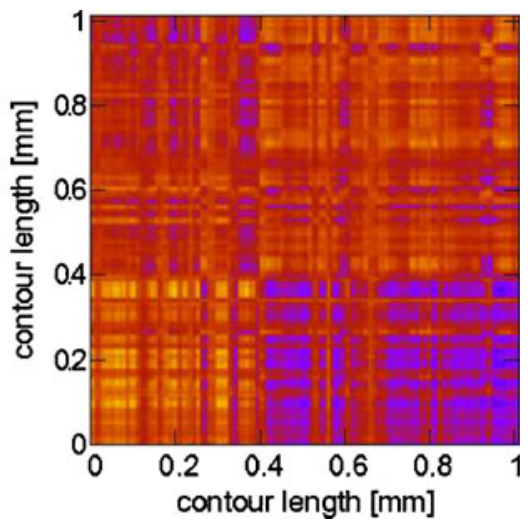

(c)
Fig. 9 Cross-proximity maps of chromosomes after $120 \mathrm{~s}$ of decondensation during interphase. The $x$-axis and the $y$-axis of the maps correspond to the contours of two distinct chromosomes, respectively. a A self-avoiding chain polymer without internal bending rigidity, $\mathbf{b}$ a self-avoiding chain polymer with a persistence length $L_{p}=300 \mathrm{~nm}$, and c a phantom chain polymer with $L_{p}=300 \mathrm{~nm}$. Blue: negative spatial correlation, yellow: positive spatial correlation 
Simulated confocal images

The three-dimensional distribution of chromatin in the cell can be determined in a confocal laser scanning microscope using fluorescent histones. The excitation by the laser can be approximated by a Gaussian intensity profile: Focused onto a center point $\vec{r}_{c}=\left(x_{c}, y_{c}, z_{c}\right)$, the intensity at an arbitrary point $\vec{r}=(x, y, z)$ is

$I_{\operatorname{exc}}\left(\vec{r}, \vec{r}_{c}\right) \propto \exp \left(-2 \frac{\left(x-x_{c}\right)^{2}+\left(y-y_{c}\right)^{2}}{w_{x y}^{2}}-2 \frac{\left(z-z_{c}\right)^{2}}{w_{z}^{2}}\right)$,

where $w_{x y}$ and $w_{z}$ are the lateral and horizontal widths of the excitation profile, respectively.

The detected fluorescence emission intensity at a particular point is in first approximation proportional to the excitation intensity in the same spot, and therefore, the Gaussian profile also holds for the emission intensity. A two-dimensional image is obtained by scanning through a plane in the sample. Each pixel of the image corresponds to the detected signal $I(\vec{r})$ at a particular position $\vec{r}$, which is contributed by all excited fluorophores: $I(\vec{r})=\sum_{i} I_{i}(\vec{r})$, where $I_{i}(\vec{r}) \propto I_{\mathrm{exc}}\left(\vec{r}, \vec{r}_{i}\right)$ is the signal intensity contributed by a single fluorophore at $\vec{r}_{i}=\left(x_{i}, y_{i}, z_{i}\right)$.

We have simulated the scan through the nucleus in the $x y$-plane at $z=0$ of the simulation lattice. At each site belonging to the chromatin chain, a virtual fluorophore was placed. For the computation of the image, we used the typical resolution of a confocal scanning microscope, $w_{x y}=$ $250 \mathrm{~nm}$ lateral and $w_{z}=1,500 \mathrm{~nm}$ horizontal. The intensities were normalized with the maximum intensity, which we detect when all lattice sites in the focus are labeled with fluorophores. To quantify the chromatin density distribution contained in the images, we computed the image cross-correlation (Görisch et al. 2005b)

$G(r)=\frac{\langle I(x+\Delta x, y+\Delta y, 0) I(x, y, 0)\rangle}{\langle I(x, y, 0)\rangle^{2}}-1$,

where $r=\sqrt{\Delta x^{2}+\Delta y^{2}}$.
The upper row of Fig. 10 illustrates the size of the laser focus (indicated by the ellipse with semi-minor axis $w_{x y}$ and semi-major axis $w_{z}$ ) in comparison to the size of the three chromosomes from Fig. 5a-c. Each of the subfigures shows a number of projections (bright gray domain) of one of these chromosomes onto the $x z$-plane. The single projections are shifted from each other by the lattice period $(1.6 \mu \mathrm{m}$, indicated by the black grid). One projection in the center is highlighted in dark gray. Corresponding simulated confocal images that are obtained by scanning across the $x y$-plane of the lattice are shown in the lower row of Fig. 10. The widely unfolded chromosomes of model A and model C occupy the nuclear space very homogeneously. They intermingle with neighboring chromosomes such that fluctuations in chromatin density are small. In contrast to that, the still rather condensed model $\mathrm{B}$ chromosome leaves portions of the nuclear space with diameters up to $1 \mu \mathrm{m}$ chromatin-free. Due to that, the laser can be focused onto certain spots at which either low or high fluorescence intensity is detectable. The image cross-correlation curves plotted in Fig. 11 express this fact quantitatively: The cross-correlation $G(r)$ of model A and C hardly set themselves apart from the background signal, while the intensity for model $\mathrm{C}$ shows a considerable decay according to the size of the chromosome. Roughly estimated, the correlation decays at about $0.5 \mu \mathrm{m}$, and the anti-correlation maximum occurs at $1 \mu \mathrm{m}$. However, the correlation length of $1.16 \mu$ found earlier by Görisch et al. (2005b) makes clear that chromatin agglomerations in the nucleus are significantly larger than the average-sized chromosomes simulated here, which implies that chromosomes are not homogeneously distributed within the nucleus.

\section{Diffusion of tracer molecules}

\section{What affects molecular diffusion?}

We have simulated the diffusion of molecules in the previously created interphase chromatin network to investigate the effects of chromatin dynamics and diffusant crowding on the molecular transport in the interphase cell nucleus. Recent 


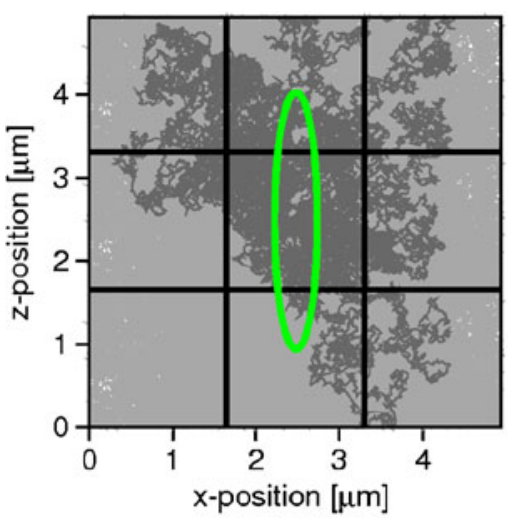

(a)

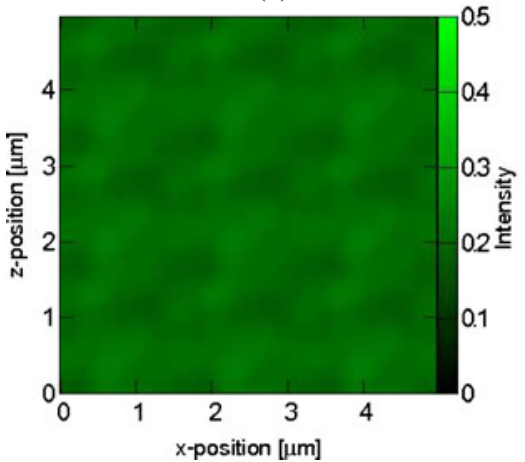

(d)

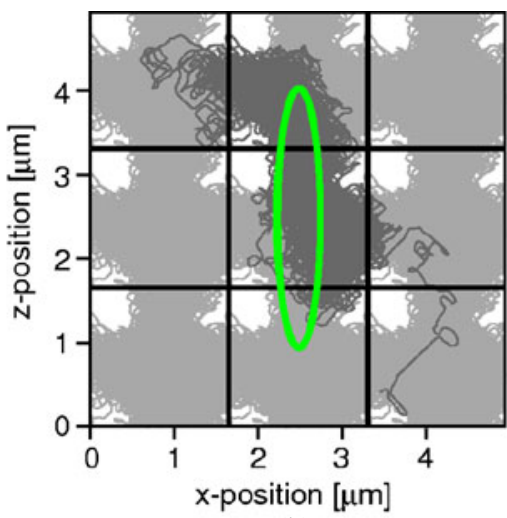

(b)

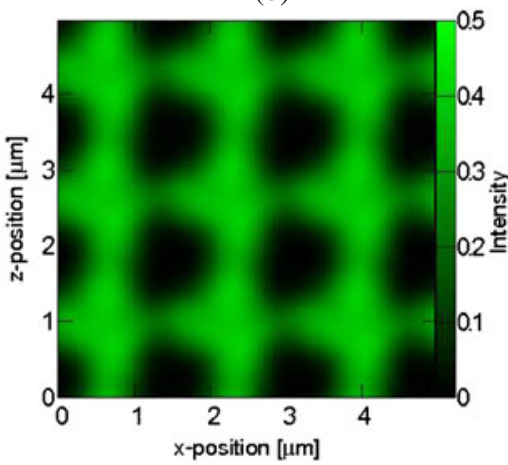

(e)

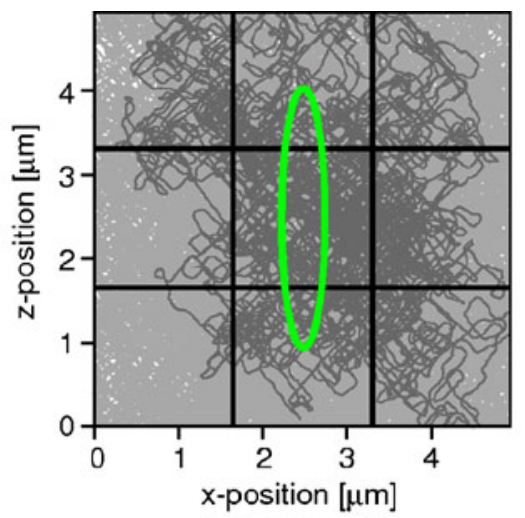

(c)

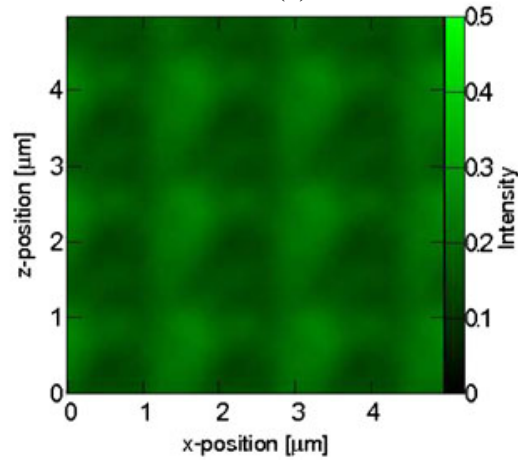

(f)
Fig. 10 Simulated confocal laser scanning. The subfigures in the upper row show the size of the laser focus in comparison to projections of the simulated chromosomes onto the $x z$-plane of the lattice (dark gray domain). The bright gray domains are periodic continuations of the black domain across the $x z$-plane of the simulation lattice. The grid indicates the periodicity. The subfigures in the lower row show confocal images obtained by scanning across the $x y$-plane of the simulation lattice. Subfigures a and $\mathbf{c}$ correspond to a self-avoiding chain polymer without internal bending rigidity, $\mathbf{b}$ and $\mathbf{d}$ correspond to a self-avoiding chain polymer with a persistence length $l_{p}=300 \mathrm{~nm}$, and subfigures $\mathbf{c}$ and e correspond a phantom chain polymer with $l_{p}=300 \mathrm{~nm}$

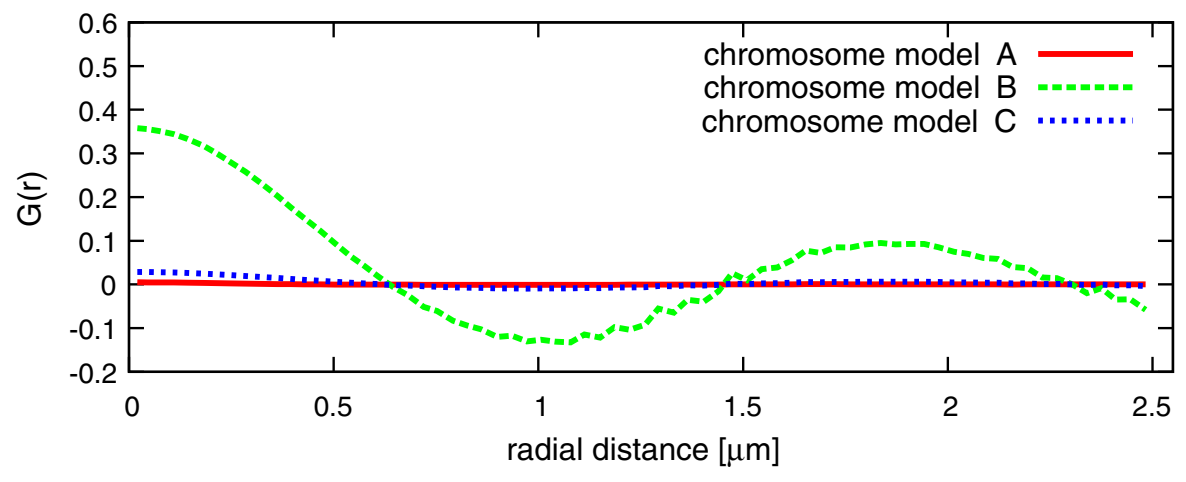

Fig. 11 Image cross-correlation $G(r)$ from simulated confocal images of chromosomes after $120 \mathrm{~s}$ of decondensation during interphase. Red solid line: self-avoiding chain polymer without internal bending rigidity (model A), green dashed line: self-avoiding chain polymer with a persistence length $L_{p}=300 \mathrm{~nm}$ (model B), blue dotted line: phantom chain polymer with $L_{p}=300 \mathrm{~nm}$ (model C). The corresponding confocal images are shown in Fig. 10 
simulation studies suggest that the diffusion behavior of small molecules with radii up to $10 \mathrm{~nm}$ does not differ between a dynamic and a static chromatin network (Wedemeier et al. 2009) and also that the influence of different network topologies cannot be resolved (Fritsch and Langowski 2010). Nuclear dynamics can only be probed with tracer molecules that are significantly influenced by the macromolecular network in the nucleus.

In simulations of molecules with radii less than $58 \mathrm{~nm}$, we observed no significant trapping in

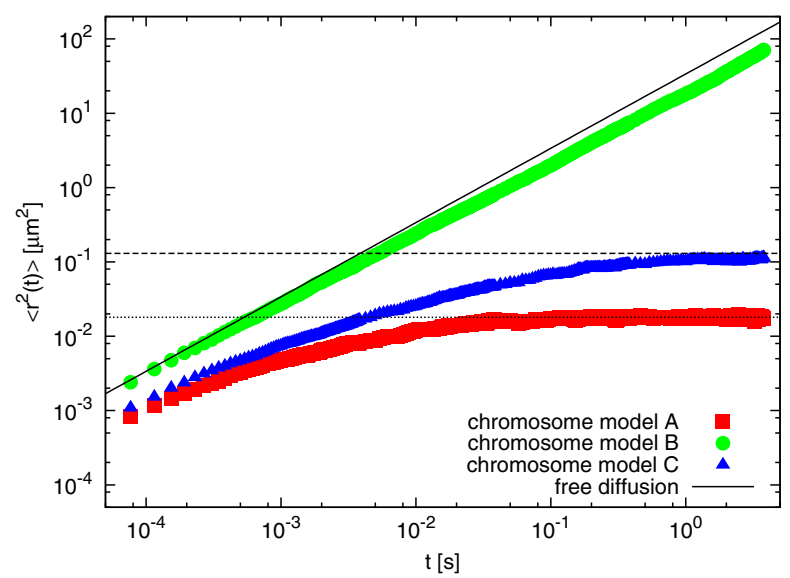

(a)

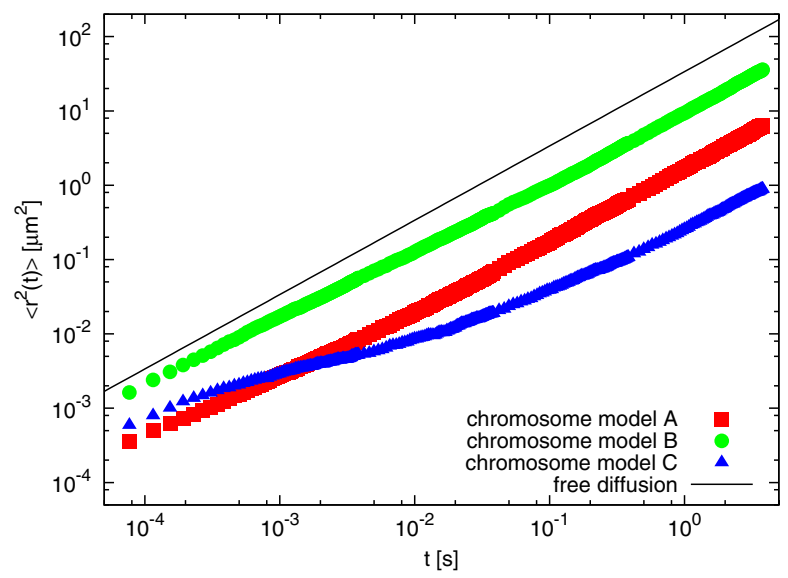

(c)

Fig. 12 Diffusion scenarios of molecules in chromatin networks. The radius of the diffusing molecule is $58 \mathrm{~nm}$. The mean squared displacements $\left\langle r^{2}(t)\right\rangle$ are plotted versus the time $t$ for a a single molecule diffusing in a static chromatin network, b crowding molecules diffusing in a static chromatin network, and $\mathbf{c}$ crowding molecules diffusing in a dynamic chromatin network. Red squares: self-avoiding static chromatin networks. In order to detect the effects of self-diffusion of the network for long times, however, we require trapping in the static network as a control. Therefore, all molecules in our diffusion simulations had the radius $R=$ $58 \mathrm{~nm}$. We investigated the following scenarios: a single molecule diffusing in a static chromatin network (scenario 1), 295 crowding molecules diffusing in a static chromatin network (scenario 2), and 295 crowding molecules diffusing in a dynamic chromatin network (scenario 3). Each of the diffusing molecules is represented by a quasi-

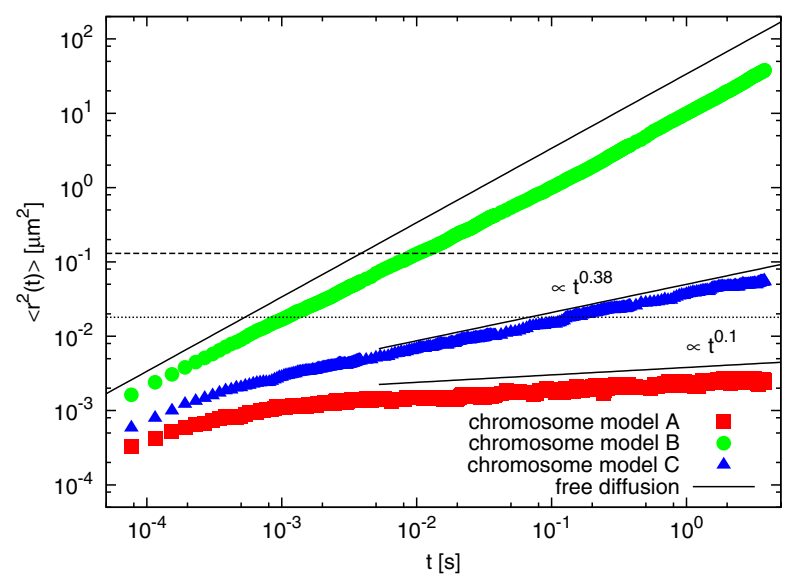

(b) chain polymer without internal bending rigidity (model A), green circles: self-avoiding chain polymer with a persistence length $L_{p}=300 \mathrm{~nm}$ (model B), blue triangles: phantom chain polymer with $L_{p}=300 \mathrm{~nm}$ (model C). The dashed and dotted lines signify the maximum MSD of confined molecules in the static network 
spherical lattice body consisting of 33 single sites, and altogether they occupy $10 \%$ of the lattice. For comparison, chromatin occupies $20 \%$ of the lattice sites in the networks of models $\mathrm{A}$ and $\mathrm{B}$ and around $18 \%$ in the networks of model $\mathrm{C}$. The slightly lower percentage in model $\mathrm{C}$ networks is due to some double occupations of sites by monomers. The monomers of the chromatin chains (radius $R_{0}=18 \mathrm{~nm}$ ) and the diffusing molecules differ in size $\left(R / R_{0}=3.2\right)$. According to "Conversion of lattice units to physical units", we adjusted the MC procedure such that one monomer performed 3.2 MC steps per MC diffusant step on average.

The fact that the diffusing molecules in the dynamic crowding scenario are larger than the monomers of the chromatin fibers prevents the formation of depletion zones around the chromatin fibers. Depletion forces are entropic forces that cause attraction between large molecules in the presence of many small molecules (Waigh 2007). If the larger molecules are very close to each other, the overall volume which the small molecules are excluded from is reduced. In turn, the gain of accessible volume increases the entropy of the small molecules and renders those states of the system more favorable in which larger molecules are generally very close. Effectively, this results in a force clustering the larger molecules. In our simulations, however, the diffusing crowder molecules are the larger ones, and consequently, no depletion attraction within the chromatin fibers occurs.

In Fig. 12, the time-dependent MSD in the different diffusion scenarios are plotted for each of the chromatin models. In panel a, the MSD curves of scenario 1 show the influence of chromosome folding on diffusional obstruction. While models $\mathrm{A}$ and $\mathrm{C}$ chromosomes contain pockets in which the diffusing molecules can be trapped, the condensed model B chromosomes do not open up wide enough. As a consequence, diffusing molecules cannot enter the interior of the chromosome and simply "bounce off". The chromosome is sensed as a smooth, easily avoidable obstacle, and the diffusion coefficient $D=\left\langle r^{2}\right\rangle /(6 t)$ (measured at long times $t$ ) is reduced to 0.58 times that of free diffusion. Furthermore, the comparison of the MSD in scenarios 2 and 3 (Fig. 12b, c) shows that molecular diffusion in the model B network is unaffected by the dynamics of the chromosome. Consequently, measuring the diffusion of small probes cannot reveal structural and dynamic properties of condensed chromosomes. The dynamics of the models $\mathrm{A}$ and $\mathrm{C}$ chromosomes, however, enable particles to evade the trapping chromatin meshes on longer time scales.

\section{Diffusant crowding}

The presence of crowders reduces the mobility of the single diffusing molecule. For instance, diffusant crowding in a static model B network reduces the diffusion coefficient $D$ to a value of 0.28 of the free diffusion coefficient $D_{0}$ (network only: reduction to $0.57 D_{0}$; see Fig. 13). The transition from anomalous diffusion to normal diffusion can be calculated as the intersection of the intermediate-time and long-time regime asymptotics of the MSD (Saxton 1994). In the scenarios 1 and 2, this transition occurs at about

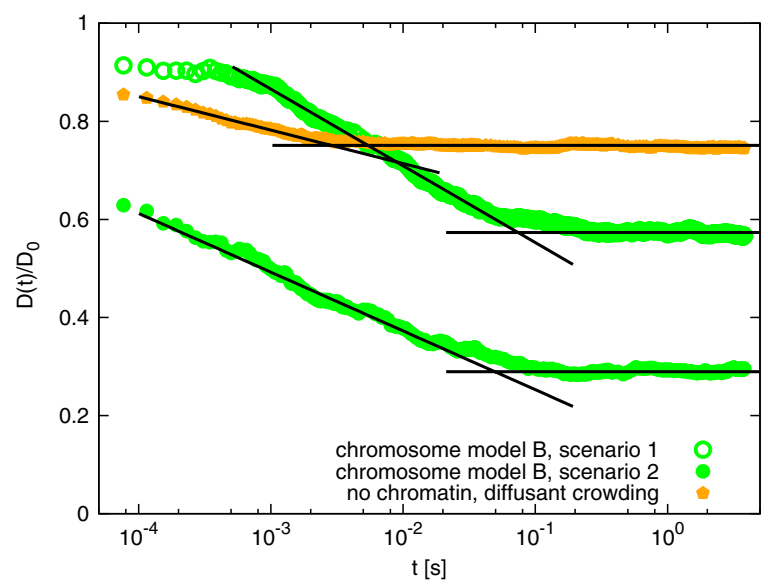

Fig. 13 Transition from anomalous diffusion to normal diffusion. The curves display the dropping of the reduced diffusion coefficients $D(t) / D_{0}$ to their long-time (macroscopic) values. Black lines indicate the intermediate time (anomalous) and long time (normal) diffusion regime. Empty green circles: a single diffusing molecule in the static network of the self-avoiding chain polymer with a persistence length $L_{p}=300 \mathrm{~nm}$ (model B and scenario 1), full green circles: crowding diffusing molecule in the static network of the self-avoiding chain polymer with a persistence length $L_{p}=300 \mathrm{~nm}$ (model B and scenario 2), orange pentagons: only crowding diffusants, no chromatin network 
0.08 and $0.05 \mathrm{~s}$, respectively, while in a crowding scenario with the same fraction of crowders but without a network it occurs already at $0.002 \mathrm{~s}$. In the static crowding scenario of models $\mathrm{A}$ and $\mathrm{C}$ chromosomes, where diffusion is anomalous at long times due to trapping (Fig. 12a), diffusant crowding skews the plateau of the MSD and hides the effect of confinement (Fig. 12b).

Depending on the degree of crowding, diffusing molecules encounter obstacles in specific average time intervals. The denser the packing, the shorter the intervals. As long as the space is not fully packed with crowding molecules, an individual molecule feels no hindrance, at least for very short times. At later times, when encountering an obstacle, the molecule is reflected. In terms of random walks on the lattice, this means that the six possible directions into which the molecule can principally displace no longer have the same probabilities; backward becomes more likely than forward. Consequently, directions of the molecule's motion at points that are separated by the characteristic encounter time are likely to be opposite. This is formalized by the velocity auto-correlation function $C_{v v}(t)=\langle\vec{v}(t) \vec{v}(0)\rangle /\left\langle\vec{v}^{2}(t)\right\rangle$. Here $\vec{v}(t)$ is the velocity of the molecule at time $t . C_{v v}(t)$ expresses the temporal decay of directional correlations in a molecular trajectory and is related to the MSD via (Jacobs and Nakanishi 1990)

$$
\langle\vec{v}(t) \vec{v}(0)\rangle=\frac{1}{2} \frac{d^{2}}{d t^{2}}\left\langle r^{2}(t)\right\rangle .
$$

Figure 14 shows the velocity auto-correlation functions corresponding to the reduced diffusion coefficients in Fig. 13. Generally, $C_{v v}(t)$ equals 1 at lag time $t=0$ and approaches zero at late lag times. The crossing of the $t$-axis toward negative values signifies the turning back of the diffusing molecules at the encounter of an obstacle. The downward amplitudes of $C_{v v}(t)$ in the scenarios with multiple crowders (solid green and orange lines in Fig. 14) are more pronounced than in the scenario with the single diffusing molecule (dot-dashed green line). In the former scenarios, the characteristic encounter time that can be read off from Fig. 14 is $\approx 0.5 \mathrm{~ms}$. In the latter, the strongest directional anticorrelation occurs rather at $\approx 1.25 \mathrm{~ms}$. Further, the auto-correlation

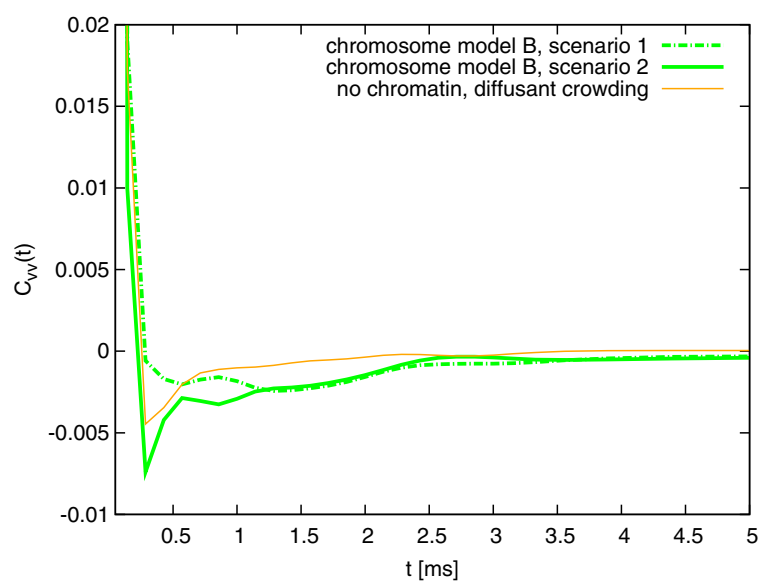

Fig. 14 Velocity auto-correlation of diffusing molecules vs. the lag time. The shown curves are smooth interpolations of numerically obtained functions $C_{v v}(t)$. Dot-dashed green line: a single diffusing molecule in the static network of the self-avoiding chain polymer with a persistence length $L_{p}=300 \mathrm{~nm}$ (model B and scenario 1), solid green line: crowding diffusing molecule in the static network of the self-avoiding chain polymer with a persistence length $L_{p}=$ $300 \mathrm{~nm}$ (model B and scenario 2), solid orange line: only crowding diffusants, no chromatin network

functions of the two scenarios containing chromatin networks (dot-dashed and solid green lines) mostly collapse at lag times later than $\approx 1 \mathrm{~ms}$. This suggests that the presence of multiple highly mobile crowders in macromolecular networks is detectable only at short lag times. At later times, the effect of the mobile crowders on the trajectory of an individual molecule cannot be distinguished from that of the network.

Chromatin dynamics influences the diffusion of molecules, and at the same time, the motion of chromatin itself is affected by the presence of crowding diffusants. In Fig. 15, the time-dependent MSD of the monomer relaxation modes are plotted for scenarios 3 and diffusant-free chromosome dynamics. Note that the relaxation of the now decondensed chromosomes is precisely Rouse-like $\left(\left\langle r^{2}(t)\right\rangle \propto t^{0.5}\right)$, other than during the previous decondensation after metaphase. For the crowder occupation of $10 \%$ we used here, we did not detect a strong influence of diffusant crowding. For instance, the monomer relaxation mode of model $\mathrm{A}$ chromosomes with diffusant crowding is reduced by a factor of less than 1.1 compared to the case without crowding 


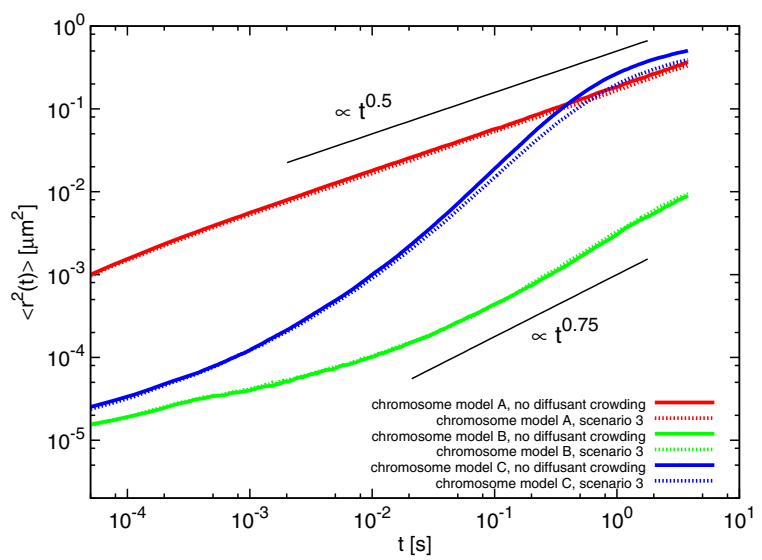

Fig. 15 Moderate deceleration of chromatin dynamics by diffusant crowding. The curves show the MSD of the monomer relaxation modes during the diffusion of crowding molecules. Red curves: self-avoiding chain polymer without internal bending rigidity (model A), green curves: self-avoiding chain polymer with a persistence length $L_{p}=$ $300 \mathrm{~nm}$ (model B). Solid curves: diffusant-free dynamics, dotted curves: diffusant crowding (scenario 3 )

diffusants. For model $\mathrm{C}$ chromosomes, both cases are hardly distinguishable. Only in model C networks does diffusant crowding reduce the long time dynamics.

\section{Nuclear elasticity}

The diffusion behavior of the molecules reflects the viscoelastic properties of the chromatin networks. The fluctuation dissipation theorem says that a system at thermal equilibrium responds to small perturbations the same way as to spontaneous thermal fluctuations. This allows to relate the fluctuation spectrum of the MSD of diffusing particles to the viscoelastic response of the system. A technique utilizing this is microrheology. Here probes are injected into a system and then the motion of the probes is recorded with a tracking technique. In this sense, our diffusion simulations are microrheological and can serve to identify the nuclear viscoelasticity induced exclusively by chromatin and crowders.

The elasticity and the viscosity of a network are formally expressed by the storage modulus $G^{\prime}(\omega)$ and the loss modulus $G^{\prime \prime}(\omega)$, respectively. $G^{\prime}(\omega)$ is the real part and $G^{\prime \prime}(\omega)$ is the imaginary part of the complex shear modulus $G^{*}(\omega)$. The Fourier transform of $G^{*}(\omega)$ is the stress relaxation modulus $G(t)$, whose Laplace transform is $\tilde{G}(s)$. $\tilde{G}(s)$ is related to the Laplace transform of the $\operatorname{MSD}\left\langle r^{2}(t)\right\rangle$, the fluctuation spectrum $\left\langle\tilde{r}^{2}(s)\right\rangle$, by $\tilde{G}(s)=k_{B} T /\left(\pi R s\left\langle\tilde{r}^{2}(s)\right\rangle\right)$. In order to get from the MSD obtained in our simulation to the moduli $G^{\prime}(\omega)$ and $G^{\prime \prime}(\omega)$, we used a method described by Mason and Weitz (1995) to avoid most of the involved Laplace and Fourier transforms: First, we computed the Laplace transform $\left\langle\tilde{r}^{2}(s)\right\rangle$ of the MSD. Then we fitted $\tilde{G}(s)$ as a function of $\left\langle\tilde{r}^{2}(s)\right\rangle$ to the numerical data to get an analytic expression. And then, by substituting $i \omega$ for $s$, we directly obtained the complex shear modulus $G^{*}(\omega)$.

The MSD in the dynamic chromatin networks (Fig. 12c) lack a plateau like in the static case (Fig. 12a). In microrheological experiments with viscoelastic media such as gels, elastic trapping can usually be detected for diffusing particles by the appearance of a trapping plateau on shorter time scales, while on longer time scales, the particles break free and diffuse normally (Biehl et al. 2004; Mason and Weitz 1995; Tseng et al. 2004; Wong et al. 2004). For our chromatin networks, no characteristic time scale for the transition from being trapped to free diffusion can be determined, suggesting that the self-diffusion of the chromatin network is too rapid to keep molecules trapped. In order to express this property in terms of viscoelasticity, $G^{\prime}(\omega)$ and $G^{\prime \prime}(\omega)$ are plotted in Fig. 16 for the case of crowding molecules diffusing in a dynamic phantom chain network (scenario 3 and model C). A viscoelastic network rapidly relaxes via viscous diffusion when sheared slowly (low frequency $\omega$ ), while it does not relax when sheared rapidly (high $\omega$ ). Therefore, one expects $G^{\prime}(\omega)>G^{\prime \prime}(\omega)$ at high frequencies and $G^{\prime}(\omega)<$ $G^{\prime \prime}(\omega)$ at low frequencies. In Fig. 16, however, the viscous modulus $G^{\prime \prime}(\omega)$ dominates on all frequencies, which means that the network is rather viscous than elastic. Tseng et al. (2004) measured a high-frequency plateau of $18 \mathrm{~Pa}$ of the storage modulus $G^{\prime}(\omega)$ in the intranuclear region of cells. This is significantly more elastic than what we obtained from our simulations. It suggests that the nucleus contains a more elastic network than can be constituted by a purely diffusive, noncrosslinked chromatin fiber. 


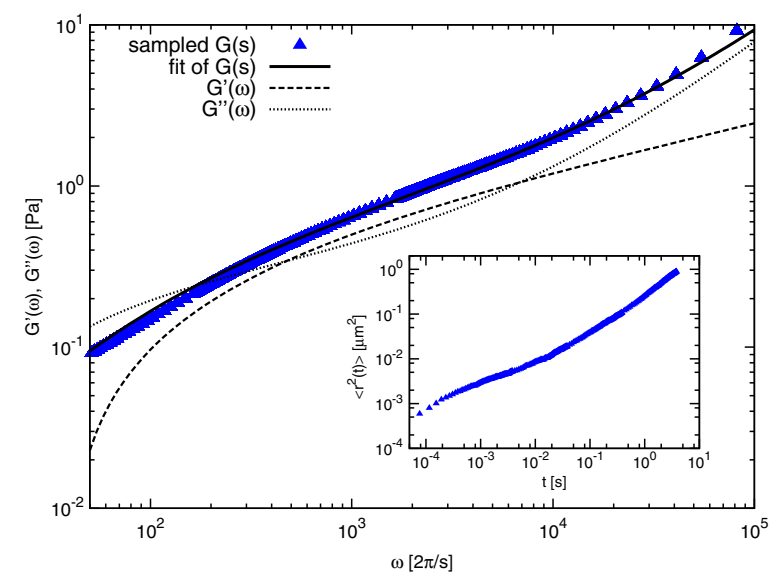

Fig. 16 Viscoelasticity of a chromatin network. The curves are computed from the diffusion of crowding molecules with a radius of $58 \mathrm{~nm}$ in a phantom chromosome network. Solid line: storage modulus $G^{\prime}(\omega)$, dashed line: loss modulus $G^{\prime \prime}(\omega)$. The inset shows the corresponding mean square displacement

\section{Discussion and conclusion}

In this simulation study, we have investigated the dynamics of chromosomes during the decondensation at the beginning of interphase. The motion of the chromosomes is assumed purely diffusive without any active contribution from, e.g., molecular motors. Three different models of polymer chain behavior were considered: model A, a highly flexible and self-avoiding chromatin fiber; model B, a persistent (i.e., stiff) and selfavoiding chromatin fiber; and model $\mathrm{C}$, a persistent and self-penetrating chromatin fiber. Model A might be viewed as a model for active, decondensed chromatin, model B for compacted chromatin and model $\mathrm{C}$ as mimicking the action of type II topoisomerases, which allow DNA chains to cross. We found that model $\mathrm{A}$ and model $\mathrm{C}$ chromosomes rapidly unfold and lose their initial metaphase-like shape. After around $120 \mathrm{~s}$, they occupy the nuclear space very homogeneously, such that almost no spatial correlations would be detectable in a confocal laser scanning microscope. Furthermore, we illustrated with crossproximity maps that these kinds of chromosomes tend to intermingle with neighboring chromosomes, such that numerous inter-chromosomal contacts occur. The auto-proximity maps show that in model $\mathrm{C}$ chromosomes, many intra- chromosomal contacts occur between genomically very distant sites, while model A chromosomes are rather compartmentalized. The dynamics of the persistent self-avoiding chromosomes (model B), however, are much slower due to selfentanglement. After $120 \mathrm{~s}$, they would still be resolvable in light microscopy. As discussed earlier by Rosa and Everaers (2008), the formation of chromatin territories can be caused by topological constraints which apply during the decondensation. Comparing the relaxation of models $\mathrm{A}$ and B chromosomes, we infer that the high persistence of chromatin amplifies these constraints and favors self-entanglement. We may also conclude that efficient daughter chromosome separation requires type II topoisomerases, as postulated earlier by Sikorav and Jannink (1994), at least for the compact and stiff regions.

After the decondensation of the chromosomes, we have simulated the diffusion of molecules in the resulting dynamic chromatin network. We found that crowding of the diffusing molecules leaves the diffusion behavior qualitatively unchanged, only reducing the long-time diffusion coefficient. Diffusant crowding causes subdiffusive anomaly $\left(\left\langle r^{2}(t) \propto t^{\alpha}\right\rangle, \alpha<1\right)$ only on very short time scales, whereas significant mesoscopic anomaly results from the obstruction by the network. In confined diffusion, crowding delays the plateau $(\alpha=0)$ of the mean squared displacement, and a low but positive anomaly parameter $\alpha$ is detected instead. Consequently, diffusant crowding causes anomaly on time scales that are shorter than those on which the obstructive network, either dynamic or static, causes anomaly.

The monomer relaxation modes of the chromosomes are only slightly slowed down in the presence of crowding diffusants at a concentration of $10 \%$. This underlines the requirement of chromatin entanglement for territory formation, since crowding by diffusants is presumably insufficient to keep chromosomes from decondensing.

For the diffusion in environments of the rather unfolded, persistent self-avoiding chromosomes (model B), the mean squared displacement of the probe molecules did not differ between the static and dynamic networks. Consequently, their dynamic properties cannot be distinguished by diffusion experiments. In particular, the diffusion 
did not reveal viscoelastic properties. In contrast, introducing dynamics to the well-decondensed $\mathrm{A}$ and $\mathrm{C}$ type chromosomes clearly facilitated the motion of diffusing molecules. The fact that we did not detect an elastic trapping regime in their mean squared displacement curves indicates that our chromatin networks are far less stiff than measured in microrheological experiments (Tseng et al. 2004). In order to provide elastic trapping, the network would have to be more rigid and more entangled, like a model B network but at the same time homogeneously distributed. If inhomogeneously distributed, chromatin can leave vast voids in the nuclear space, and diffusing molecules are likely to travel on these voids rather than within entangled network meshes. As we have found, a homogeneous distribution is in turn prevented by the entanglement, which inhibits the unfolding from compact metaphase conformations. To gain high elasticity, chromatin may change its viscoelastic properties during interphase in such a way that it can rapidly decondense and then consolidate. This could be effected, e.g., by crosslinks that induce the formation of chromatin loops (Bohn et al. 2007; Cremer et al. 1996; Lieberman-Aiden et al. 2009; Münkel and Langowski 1998). In addition to chromatin, then, one might need to invoke contributions by another, stiffer macromolecular network to account for the high elasticity of the nucleus. Such a network might also affect the folding of chromosomes.

Summing up, the key to understanding the dynamics of interphase chromosomes is their viscoelasticity. The simulations described here encourage future investigations that target the dynamic crosslinking of chromatin in order to determine to what extent chromatin is responsible for the nuclear elasticity.

\section{References}

Bancaud A, Huet S, Daigle N, Mozziconacci J, Beaudouin J, Ellenberg J (2009) Molecular crowding affects diffusion and binding of nuclear proteins in heterochromatin and reveals the fractal organization of chromatin. EMBO J 28(24):3785-3798

Biehl R, Guo X, Prud'homme RK, Monkenbusch M, Allgeier J, Richter D (2004) Diffusion of compact macromolecules through polymer meshes: mesh dynamics and probe dynamics. Phys B Condens Matter 350(1-3):76-78 (Proceedings of the third European conference on neutron scattering)

Bohn M, Heermann DW, van Driel R (2007) Random loop model for long polymers. Phys Rev E (Statistical, Nonlinear, and Soft Matter Physics) 76(5):051805

Carmesin I, Kremer K (1988) The bond fluctuation method: a new effective algorithm for the dynamics of polymers in all spatial dimensions. Macromolecules 21:2819-2823

Cremer C, Münkel Ch, Granzow M, Jauch A, Dietzel S, Eils R, Guan XY, Meltzer PS, Trent JM, Langowski J, Cremer T (1996) Nuclear architecture and the induction of chromosomal aberrations. Mutat Res Rev Genet Toxicol 366(2):97-116

Dix JA, Verkman AS (2008) Crowding effects on diffusion in solutions and cells. Annu Rev Biophys 37:247-263

Doi M, Edwards SF (1986) The theory of polymer dynamics. Clarendon, Oxford

Dross N, Spriet C, Zwerger M, Müller G, Waldeck W, Langowski J (2009) Mapping eGFP oligomer mobility in living cell nuclei. PLOS ONE 4(4):e5041, 04

Ellis R, Minton A (2003) Cell biology: join the crowd. Nature 425:27-28

Farge E, Maggs AC (1993) Dynamic scattering from semiflexible polymers. Macromolecules 26(19):50415044

Fritsch CC, Langowski J (2010) Anomalous diffusion in the interphase cell nucleus: the effect of spatial correlations of chromatin. J Chem Phys 133:025101

Görisch SM, Wachsmuth M, Ittrich C, Bacher CP, Rippe K, Lichter P (2004) Nuclear body movement is determined by chromatin accessibility and dynamics. Proc Natl Acad Sci U S A 101:13221-13226

Görisch SM, Lichter P, Rippe K (2005a) Mobility of multisubunit complexes in the nucleus: accessibility and dynamics of chromatin subcompartments. Histochem Cell Biol 123(3):217-228

Görisch SM, Wachsmuth M, Toth KF, Lichter P, Rippe K (2005b) Histone acetylation increases chromatin accessibility. J Cell Sci 118(24):5825-5834

Guigas G, Weiss M (2008) Sampling the cell with anomalous diffusion - the discovery of slowness. Biophys $\mathrm{J}$ 94(1):90-94

Jacobs D, Nakanishi H (1990) Autocorrelation functions for discrete random walks on disordered lattice. Phys Rev A 41(2):706-719

Klar TA, Hell SW (1999) Subdiffraction resolution in farfield fluorescence microscopy. Opt Lett 24:954-956

Lieberman-Aiden E, van Berkum NL, Williams L, Imakaev M, Ragoczy T, Telling A, Amit I, Lajoie BR, Sabo PJ, Dorschner MO, Sandstrom R, Bernstein B, Bender MA, Groudine M, Gnirke A, Stamatoyannopoulos J, Mirny LA, Lander ES, Dekker J (2009) Comprehensive mapping of longrange interactions reveals folding principles of the human genome. Science 326(5950):289-293

Mason TG, Weitz DA (1995) Optical measurements of frequency-dependent linear viscoelastic moduli of complex fluids. Phys Rev Lett 74(7):1250-1253 
Metropolis N, Rosenbluth AW, Rosenbluth MN, Teller AH, Teller E (1953) Equation of state calculations by fast computing machines. J Chem Phys 21:10871092

Metzler R, Klafter J (2000) The random walk's guide to anomalous diffusion: a fractional dynamics approach. Phys Rep 339:1-77

Müller WG, Walker D, Hager GL, Mcnally JG (2001) Large-scale chromatin decondensation and recondensation regulated by transcription from a natural promoter. J Cell Biol 154(1):33-48

Münkel C, Langowski J (1998) Chromosome structure predicted a by polymer model. Phys Rev E 57(5):58885896

Politz JC, Tuft RA, Pederson T (2003) Diffusion-based transport of nascent ribosomes in the nucleus. Mol Biol Cell 14(12):4805-4812

Rosa A, Everaers R (2008) Structure and dynamics of interphase chromosomes. PLoS Comput Biol 4(8):e1000153

Rubinstein M, Colby RH (2003) Polymer physics. Oxford University Press, Oxford

Saxton MJ (1994) Anomalous diffusion due to obstacles: a Monte Carlo study. Biophys J 66(2, Part 1):394401

Siebrasse JP, Kubitscheck U (2009) Single molecule tracking for studying nucleocytoplasmic transport and intranuclear dynamics. Methods Mol Biol 464:343-61

Sikorav JL, Jannink G (1994) Kinetics of chromosome condensation in the presence of topoisomerases-a phantom chain model. Biophys J 66(3):827-837
Tseng Y, Lee J, Kole T, Jiang I, Wirtz D (2004) Microorganization and visco-elasticity of the interphase nucleus revealed by particle nanotracking. J Cell Sci 117:2159-2167

Wachsmuth M, Waldeck W, Langowski J (2000) Anomalous diffusion of fluorescent probes inside living cell nuclei investigated by spatially-resolved fluorescence correlation spectroscopy. J Mol Biol 298(4):677-689, 05

Waigh TA (2007) Applied biophysics. Wiley, Chichester

Wedemeier A, Merlitz H, Wu C-X, Langowski J (2007) Modeling diffusional transport in the interphase cell nucleus. J Chem Phys 127(4):045102

Wedemeier A, Zhang T, Merlitz H, Wu C-X, Langowski $\mathrm{J}$ (2008) The role of chromatin conformations in diffusional transport of chromatin-binding proteins: Cartesian lattice simulations. J Chem Phys 128(15): 155101

Wedemeier A, Merlitz H, Wu C-X, Langowski J (2009) How proteins squeeze through polymer networks: a Cartesian lattice study. J Chem Phys 131(6):064905

Weidemann T, Wachsmuth M, Knoch TA, Müller G, Waldeck W, Langowski J (2003) Counting nucleosomes in living cells with a combination of fluorescence correlation spectroscopy and confocal imaging. J Mol Biol 334(2):229-240

Wong IY, MGardel L, DReichman R, Weeks ER, Valentine MT, Bausch AR, Weitz DA (2004) Anomalous diffusion probes microstructure dynamics of entangled F-actin networks. Phys Rev Lett 92(17):178101 